\title{
On Multi-Fluid models for spray-resolved LES of reacting jets
}

\author{
F. Doisneau ${ }^{\mathrm{a}, *}$, M. Arienti ${ }^{\mathrm{a}}, \mathrm{J}$. Oefelein $^{\mathrm{a}}$ \\ ${ }^{a}$ Combustion Research Facility, Sandia National Laboratories, Livermore, CA 94551-0969, USA
}

\begin{abstract}
Numerical simulation of sprays can potentially be used for assessing the performance and variability of engines. But today's simulations only provide average quantities, after calibration. Spray injection can be thought of as a multiscale problem with 4 levels (chamber, mixing layer, drop scale, and nozzle). Because of their complex interactions, increasing predictivity requires simulations to capture more of these scales. To assess the trade-offs in this regard, we perform a review of recent Diesel spray simulations. After highlighting the importance of the mixing layer in driving spray dynamics, we analyze various two-phase flow formalisms and show the potential benefits of a Eulerian spray formulation combined with Large Eddy Simulation (LES) to capture a variable-density mixing layer (VDML). We then present a framework and numerical methods to make this description operative and show how it performs on a realistic autoignition case called Spray A.
\end{abstract}

Keywords: Atomization; Dense spray; Mixing Layer; Spray combustion; Large Eddy Simulation.

\section{Introduction}

Predicting spray injection, atomization, and combustion is required for many engineering problems. In piston engines, adjusting the injection sequence is considered a viable way to improve efficiency while reducing pollutants. However, the injection process is multiscale, with a ratio of $10^{3}$ between the injector nozzle diameter and the burning plume length. Even smaller turbulence scales exist in the nozzle flow. Despite the increase in computational power, representing all these scales, together with the physics of compressibility, turbulence, entrainment, atomization, spray transport, phase change, mixing, and turbulent combustion, still involves many challenges.

Spray injection can be thought of as a multiscale problem with 4 levels, with ranges that depend on a characteristic length and Reynolds number:

- Chamber: $L=O\left(10^{-1} \mathrm{~m}\right)$ with $\operatorname{Re}_{c}=O\left(10^{5}\right)$,

- Mixing layer: $d_{i n j}=O\left(10^{-4} \mathrm{~m}\right)$ with $\operatorname{Re}_{\mathrm{ML}}=O\left(10^{3}\right)$,

- Droplet wake: $d_{d r o p}=O\left(10^{-5} \mathrm{~m}\right)$ with $\operatorname{Re}_{p}<10^{3}$,

- Nozzle flow: $d_{i n j}$ with $\operatorname{Re}_{d}=O\left(10^{5}\right)$.

On one hand, high-fidelity simulations of the near-nozzle region with interface capturing methods [1-4] have allowed a better understanding of atomization, but they consume significant computational and human resources

\footnotetext{
${ }^{*}$ Corresponding author

Email address: fdoisne@sandia.gov (F. Doisneau)
}

and do not allow one to study wide domains, combustion, or entrainment. On the other hand, simulations that capture the chamber scales (swirl, tumble and their turbulence), possibly without accounting for injection, have been examined [5-8]. This methodology has been naturally extended to applied problems using ReynoldsAverage Navier-Stokes (RANS) [9] or coarse Large Eddy Simulation (LES) [10]. Related numerical studies have shown good agreement on steady, average, and large scale features, namely the liquid length, vapor penetration, cone angle, and region of combustion. Such "chamberscale" studies are conducted using multiphysics codes with coarse meshes and calibrated subgrid models (see a review in Section 2.1). However, the subgrid models are adapted from the single-phase flow literature, eventually with an ad hoc treatment for spray break-up. In contrast, in classical atomization and supercritical jets, turbulence in the mixing layer is strongly modified by large variations in density. We refer to this as the Variable-Density Mixing Layer (VDML) and we note that current subgrid models do not describe VDML well. So the current subgrid models make the overall "chamber-scale" approach poorly predictive. Ongoing research aims at modeling two-phase turbulence [11-14], potentially with phase change and chemistry. But even these advanced models are limited when the liquid mass loading becomes high; i.e., near the liquid core. In addition, the droplet scale is difficult to characterize considering the broad spectrum produced by the atom-

May 28, 2016 
ization process. So $d_{d r o p}$ is indicative but has a large variance. Progress has been made at fixed or narrow droplet scales [11-14], but there are few if any studies of the effect of the droplet wakes on the jet dynamics. Finally, nozzle turbulence is recognized as impacting injection dynamics with its intense, small scale turbulence and possible cavitation. Only recent studies have begun to address this from first principles [3, 15].

This paper fosters the idea of increasing the spatial resolution of "chamber-scale" simulations of injection to mitigate the deficiency in subgrid models. We refer to this approach as "spray-resolved." We suggest it will facilitate next-generation spray studies focused on transient, local, realization-dependent quantities of interest such as slip velocities or autoignition kernels [11,14]. But the challenges to enable spray-resolved simulations are i) a model that describes both phases accurately and is compatible with ii) a transport scheme that preserves the steep fronts and the vacuum zones, and iii) an overall integration strategy that achieves strong coupling efficiently in the context of massively-parallel computing. These requirements match the features of Euler-Euler spray modeling [16-18]. Such simulations can be costly, but can potentially be used as numerical experiments to devise next-generation subgrid models or datasets for Model Order Reduction [19, 20].

The paper is organized as follows. In Section 2 we discuss the limitations of the existing models for spray injection. We then describe the relevance of a sprayresolved strategy, for which the Euler Spray formalism is well suited. In Section 3, we present our model and discuss its merits for the simulation of liquid injection. In Section 4, results on momentum coupling in the liquid gas mixing layer and on autoignition of a Diesel jet are shown to demonstrate the potential of the new strategy.

\section{Spray-resolved LES of a jet}

In the process of injection and atomization, the local state of the liquid fuel is defined as either continuous (liquid core and ligaments) or dispersed (dense and dilute spray). The distinction is convenient though not strict since a churning liquid core, atomizing ligaments, and coalescing drops in a dense spray are difficult to theoretically characterize and experimentally identify. The classical strategies to model such a state are: Stochastic Lagrangian Spray or Direct Simulation Monte Carlo (DSMC) [21]; Eulerian Spray [22]; Discrete Particle Simulation (DPS) [23]; Particle-Resolved (PR) DPS [24]; and Eulerian Liquid $[1,3]$. Any of these models is then coupled to the carrier gas using volume sources (for the three first approaches) or boundary conditions (for the last two). With each of these approaches, although at different scales, there will be large density, momentum, and temperature gradients, as well as rapid phase changes. This makes the problem numerically challenging. To reduce the spatial and thermodynamic stiffness, some authors $[9,25]$ have replaced the Eulerian Liquid approach by an Equivalent Gas using an enlarged injector to preserve mass and momentum flow rates. But capturing these gradients is of paramount importance to render the physics of the spray since i) the transfer rates depend on the local liquid mass loading, and ii) the fronts do not smooth out with time compared to gas dynamics where acoustics and diffusion tend to bring back a balance fast. This has been stated, for instance, when resolving spray-acoustics interactions $[26,27]$. And it is expected that spray ignition, combustion, and flame extinction are very sensitive to the steepness of the droplet cloud density fronts, as suggested by Direct Numerical Simulation of droplet evaporation in turbulence [28].

Table 1 summarizes the models for transport in physical space. Models below the double line can all be derived from a kinetic equation, either the Boltzmann or WilliamsBoltzmann equations [31]. They differentiate each other by their level of compressibility and hydrodynamics. Liquid and gas can be accounted for with Navier-Stokes equations and perfect gas or real fluid thermodynamics. Kinetic modeling of a spray is foundational to all Eulerian Spray models and to DSMC. Eulerian models then trade deterministic solutions for a statistical description of local velocities at equilibrium; e.g., monokinetic or Gaussian (as discussed in Section 3). The DSMC model trades linearity and genericity of its transport part for a sublinear convergence with the number of parcels.

Knowing the origin of these models is key to understanding their capabilities and limits as well as their physical and numerical properties. DSMC and Eulerian methods have in principle no differences in terms of the physics they can describe. But we show in the following paragraph that DSMC is chosen most often for practical reasons imposed by the insufficient spatial resolution achieved in the attempts reported in the following section.

\subsection{A review of modeling strategies for Spray A}

To illustrate the spray modeling strategies, we focus on the Engine Combustion Network (ECN) Spray A case [32]. This case is representative of a Diesel injector (high chamber and injection pressures) and is welldocumented. Table 2 presents a review of Spray A computations with the choice of model for the liquid phase. Resolution $d_{i n j} / \Delta x$ is given for the first inlet cell. An inequality implies the use of Adaptive Mesh Refinement (AMR). The effect of coarsening grids on accuracy in the spray region is not discussed. 
There are several points to make related to these past studies. First, the range of mesh resolutions presented in Table 2 is strikingly broad with a ratio of length scales $\eta=240$. We can formally translate this into cost by considering that a space refinement of cell lengths $\eta$ induces an increase in cost that is $O\left(\eta^{4}\right)$ so that an interface capturing simulation would be $\eta^{4}=3 \times 10^{9}$ times more costly than the coarsest simulation if performed on the same volume. Of course this does not include that RANS is steady and can be computed on a 2D grid, whereas LES and DNS require $3 \mathrm{D}$ and the convergence of statistics through multiple realizations. Second, (PR-)DPS is not used in present calculations of an entire spray. We attribute this to the cost of such a simulation and to the difficulty of defining deterministically meaningful initial and boundary conditions. Third, there are many simulations in groups 1 and 2. Using a resolution about or below $d_{i n j}$, they correspond to "chamber-scale" simulations. They mostly involve DSMC coupled to RANS or LES. They provide experimental agreement in terms of liquid length, vapor penetration, spray angle, and region of combustion. However, the statistics are rarely discussed or validated due to cost but also due to a lack of data. Thus current "chamberscale" spray simulations appear poorly conclusive. Fourth, the large number of "chamber-scale" simulations is due to their obvious lower cost. Conversely, there are only 3 simulations in group 4. Their high-fidelity comes at high cost and prevents from running parametric analysis or converging statistics. There are surprisingly few group-3 simulations. To-date, "chamber-scale" simulations are widely accepted as engineering simulations and group-4 simulations are performed for research when resources are available, while group-3 simulations are typically disregarded because they are difficult to set-up, as will be demonstrated below, and their cost is relatively high.

The existence and the relevance of VDML scales has been challenged by authors claiming that grid convergence can be reached with $\Delta x \sim d_{i n j}$ [55]. An apparent grid convergence can indeed be observed if the scales of the chamber flow and of the VDML are sufficiently separated, but more scales have to appear at some point with refinement; e.g. the jet shear layer scales. So this observation corresponds merely to a plateau in the convergence curve. Similarly, AMR might, depending on the implementation, achieve a discretization that is still too coarse in the jet region if the AMR refinement criterion is blind to the jet itself and to VDML fluctuations. Finally, Pope's resolution criterion has been used to claim that a significant portion of the turbulent kinetic energy was captured with group-1 and group-2 simulations, but this criterion cannot be invoked if the subgrid model is not "aware" that the jet is injecting a large amount of unresolved energy and/or if the scheme is dissipative. In conclusion, group-1 and group-2 simulations are not converged with regard to the physics. They provide results that are strongly dependent on calibrated models with remarkable difficulties in quantifying the uncertainty [50]

In contrast, for group-3 simulations, the large scales of the VDML appear to be resolved as we show in Section 4. Since these scales have been identified as being crucial for mixing and badly accounted for in current subgrid models, it is thus interesting to resolve them in the frame of LES. We suggest that group-3 simulations are a missing link in current research on injection. Thus, we define simulations with a spatial resolution finer than $d_{i n j}$ as "spray-resolved LES" because of their potential to accurately describe the large scales of VDML.

\subsection{On the operating resolution of spray models}

The validities of the approaches are summarized in Fig. 1 as a function of grid resolution. Here we give numerical arguments to explain their limitations. For $\Delta x \ll$ $d_{\text {drop }}$, all the scales of the interface are accessible, by definition of a drop being the smallest liquid entity. Then, the turbulence stemming from the nozzle may not be resolved but its modeling is less challenging (except for cavitating flows). For $\Delta x>d_{d r o p}$, the liquid-gas interface is poorly resolved. Ongoing research attempts to combine interface capturing algorithms with a filtered description of the interface $[56,57]$. For applied computations however, it is perhaps better to assume that the actual interface is at the subgrid level by switching from an interface-capturing method to a mesoscopic spray model. For $\Delta x>d_{i n j}$, the VDML is no longer resolved. We define $d_{\text {dilute }}$ so that even coarser meshes with $\Delta x>d_{\text {dilute }}$ make the source terms from the entire liquid core negligible and coupling no longer stiff. In this regime, only the chamber-scale flow can actually be resolved and it is decoupled from any dynamic effect induced by the spray.

At too coarse a resolution, Eulerian models reach a limit of validity because they encounter mesh diffusion errors on the liquid mass concentration, which explains why the scarce group-2 Eulerian simulations [43, 44] use a purposely designed "downstream-injection" numerical technique, while group-1 simulations use DSMC. DSMC holds at coarse resolution thanks to its more accurate representation of the mass concentration field since the parcel trajectories are computed to machine precision. However, this description is not exempt from numerical diffusion because it exchanges momentum and enthalpy with the coarse Eulerian grid, which eventually compromises its accuracy on droplet trajectories.

For $d_{i n j} / 30<\Delta x<d_{i n j}$ mesoscopic spray models still perform well. Because of the very fine grid, DSMC 
would require an advanced coupling strategy to enforce mass, momentum, and heat transfer in cells full of liquid $\left(\Delta x<d_{i n j}\right)$. This is due to the dispersion of particles in physical space being arbitrary with respect to the Eulerian grid, which prevents one from an all-at-once time integration of the system. Finer grids, allowing more uneven and unsteady flow fields, would require more DSMC samples, inducing load-balancing issues in parallel computing [58]. In addition, the slow statistical convergence of DSMC, and the difficulty to achieve an efficient, bias-free seeding due to the strong non-linearity of the particle trajectories, dramatically increase the cost of mitigating contamination of the statistics of interest. In particular, there is a risk of triggering events such as spurious ignition due to stochastic noise. So we retain Eulerian spray approaches as good candidates to perform spray-resolved simulations.

\section{Coupled Eulerian spray model}

A spray can be described as disperse liquid structures suspended in a carrier gas using a fluid-kinetic model [31]. To do so, gas equations are strongly coupled to a kinetic equation, statistically describing the disperse phase. The internal coordinates translate the degrees of freedom of droplets and are the velocity vector, temperature, and radius $(\boldsymbol{c}, \theta, r)$. Coupling is achieved through mass, momentum, and enthalpy source terms accounting for vaporization, drag, and heat transfer in a conservative way. This description is sufficient if the volume fraction of the disperse phase is not too high. It can be closed if the liquid structures are spherical enough [22].

The fluid-kinetic system is given by

$$
\left\{\begin{array}{l}
\text { Multicomponent Navier-Stokes }+ \text { spray source terms } \\
\partial_{t} f+\partial_{x} \boldsymbol{c} f+\partial_{\boldsymbol{c}} \boldsymbol{F} f+\partial_{\theta} \mathrm{H} f+\partial_{r} \mathrm{E} f=\mathrm{B}+\mathrm{C}
\end{array}\right.
$$

where the source terms describe vaporization $(\mathrm{E})$, drag $(\boldsymbol{F})$, and heating $(\mathrm{H})$, break-up (B), and coalescence (C). The integrals defining the coupling terms in the NavierStokes equation are computed using drop closures. For clarity, we neglect B and C in the present study, keeping in mind that they have an influence on the spray dynamics and that the present framework allows such models to be easily considered. The number density function (NDF), $f(t, \boldsymbol{x} ; \boldsymbol{c}, \theta, r)$, has a high dimensional phase space (5D in addition to the $4 \mathrm{D}$ of space and time). This can be reduced by Monte-Carlo sampling, leading to DSMC.

An alternative reduction approach is based on sizeconditioning and shape-presuming [22]. It can be thought of as derived in two modeling steps: i) the velocity treatment (on a size-conditioned basis) that leads to the various models for transport in physical space described at the beginning of Section 2 and ii) the size treatment. For i), assuming perfectly correlated velocities; i.e. the monokinetic closure $f(t, \boldsymbol{x} ; \boldsymbol{c}, \theta, r) \approx$ $\kappa(t, \boldsymbol{x} ; r) \delta\left(\boldsymbol{c}-\boldsymbol{u}_{l}(t, \boldsymbol{x})\right) \delta\left(\theta-T_{l}(t, \boldsymbol{x})\right)$, leads to a Eulerian system and is valid as long as $r$ is below a critical size, above which trajectory crossings will occur. The monokinetic assumption can been relaxed, leading to models accounting for statistical trajectory crossings; e.g. caused by turbulence [29, 59]. For simplicity, we apply the monokinetic assumption, while seeking accuracy on the droplet size by discretizing the size phase space. For ii), we consider a discretization of the size variable referred to as Multi-Fluid, which is, in fact, an extension of sectional methods that include sections with independent velocity treatments [22]. The entire size distribution can be accounted for by combined moment and Multi-Fluid methods. It can be efficiently transported with arbitrary accuracy, allowing one to render the missing global size moments; e.g., the Sauter Mean Diameter. The Multi-Fluid approach can uniquely reconstruct any NDF from the knowledge of a set of moments in size, velocity, and temperature, where the number of moments $n$ is related to the number of unknown parameters in the shapes $\kappa_{k}$ that are presumed in each section $k$ [60]. Choosing two moments is typically a good trade-off [27] for polydisperse problems with diverse drop dynamics.

The Multi-Fluid modeling strategy has been applied to engine sprays [16] and specific extensions were considered $[17,61]$ in cases where the numerical setup was limiting local drop concentrations to the dilute spray regime; e.g., by injecting liquid downstream of the nozzle. The present study focuses on capturing near-nozzle scales and connecting dense spray dynamics to a dilute reacting spray. In the following and without loss of generality, we consider one section so that indices $k$ are replaced by a single section $l$. Multiple size intervals and the corresponding sets of moments can be used to describe polydispersity with higher accuracy [22, 27, 62]. This strategy is under development for the Spray A case [18]. We resort to two moments in size in the one section chosen. These are number and mass $\left(n_{l}, m_{l}\right)$. So we need to account for 6 moments in total

$$
\left(\begin{array}{c}
n_{l} \\
m_{l} \\
m_{l} \boldsymbol{u}_{l} \\
m_{l} h_{l}
\end{array}\right)(t, \boldsymbol{x})=\int_{\mathbb{R}^{+}} \int_{\mathbb{R}^{+}} \int_{\mathbb{R}^{3}}\left(\begin{array}{c}
1 \\
m(r) \\
m(r) \boldsymbol{c} \\
m(r) h(\theta)
\end{array}\right) f(t, \boldsymbol{x} ; \boldsymbol{c}, \theta, r) \mathrm{d} \boldsymbol{c} \mathrm{d} \theta \mathrm{d} r,
$$

where $m(r)=\rho_{l} \frac{4}{3} \pi r^{3}, h(\theta)=\int c_{p, l}(\theta) \mathrm{d} \theta$, and $h_{l}=h_{l}\left(T_{l}\right)$. The disperse phase is therefore described by 6 scalar Eulerian fields in 4D, instead of the initial scalar 9D problem. 
The final governing system is

$$
\left\{\begin{array}{l}
\partial_{t} \rho_{g} Y_{i}+\partial_{x} \rho_{g} Y_{i} \boldsymbol{u}_{g}=-\partial_{x} \mathbf{q}_{i}+\omega_{i}, i \in \llbracket 1 ; N_{\mathrm{sp}} \rrbracket, i \neq f \\
\partial_{t} \rho_{g} Y_{f}+\partial_{x} \rho_{g} Y_{f} \boldsymbol{u}_{g}=-\partial_{x} \mathbf{q}_{f}+\omega_{f}+\mathrm{E}_{l}^{m} \\
\partial_{t} \rho_{g} \boldsymbol{u}_{g}+\partial_{x} \rho_{g} \boldsymbol{u}_{g} \otimes \boldsymbol{u}_{g}=-\partial_{x} p-\partial_{x} \mathbf{S}-\boldsymbol{F}_{l}+\boldsymbol{u}_{l} \mathrm{E}_{l}^{m} \\
\partial_{t} \rho_{g} e_{g}+\partial_{x} \rho_{g} e_{g} \boldsymbol{u}_{g}=-p \partial_{x} \boldsymbol{u}_{g} \partial_{x} \mathbf{q}-\mathrm{H}_{l}-\boldsymbol{F}_{l} \cdot\left(\boldsymbol{u}_{g}-\boldsymbol{u}_{l}\right)+h_{l} \mathrm{E}_{l}^{m} \\
\partial_{t} n_{l}+\partial_{x} n_{l} \boldsymbol{u}_{l}=-\mathrm{E}_{l}^{n} \\
\partial_{t} m_{l}+\partial_{x} m_{l} \boldsymbol{u}_{l}=-\mathrm{E}_{l}^{m} \\
\partial_{t} m_{l} \boldsymbol{u}_{l}+\partial_{x} m_{l} \boldsymbol{u}_{l} \otimes \boldsymbol{u}_{l}=\boldsymbol{F}_{l}-\boldsymbol{u}_{l} \mathrm{E}_{l}^{m} \\
\partial_{t} m_{l} h_{l}+\partial_{x} m_{l} h_{l} \boldsymbol{u}_{l}=\mathrm{H}_{l}-h_{l} \mathrm{E}_{l}^{m},
\end{array}\right.
$$

where $N_{\mathrm{sp}}$ is the number of species, $\omega_{i}$ the chemical source for species $i$, and $f$ the index for fuel vapor. For the sake of legibility, we have omitted use of filter notation associated with LES and refer the reader to the literature for turbulent fluxes to be added in the species flux $\mathbf{q}_{i}$, stress tensor $\mathbf{S}$, and heat flux $\mathbf{q}$.

\subsection{Gas-liquid coupling models}

We assume spherical drop closures for all the transfers; i.e., Stokes drag and heat laws, and $d^{2}$ vaporization, with convective corrections. As a first step, the transfers are computed from the resolved quantities and no two-phase subgrid models were used. So the droplet transport is not influenced by the gas subgrid turbulence and the gas is not influenced by the droplets' subgrid motion. The authors agree that turbulent dispersion is important in sprays, but in this dense spray context no satisfactory model exists and the physics is not well known. Moreover the mesh resolution of the present studies may compensate for part of this.

A local characteristic drag time stems from eigenvalue analysis of the two-way coupled momentum equations

$$
\tau_{c}^{u}(t, \boldsymbol{x})=\frac{\tau^{u}\left(r_{l}(t, \boldsymbol{x})\right)}{1+m_{l}(t, \boldsymbol{x}) / \rho_{g}(t, \boldsymbol{x})}
$$

where $\tau^{u}(r)$ is the characteristic time of the drag force $\boldsymbol{F}_{l}$ and the local drop radius $r_{l}$ can be computed from $n_{l}$ and $m_{l}$. The same argument applies to a coupled heat transfer time $\tau_{c}^{T}$ by using $\tau^{T}(r)$ the characteristic time of $\mathrm{H}_{l}$. So the characteristic times for the two-way coupled system get shorter with higher liquid loading. This implies that the discrepancies introduced by spherical closures do not have a significant impact in the regions where loading is high, as suggested in Doisneau et al. [18] and verified for the liquid core. In these regions, the overall dynamics is dominated by the liquid so the coupling actually maintains the velocities and temperatures close to equilibrium.

Vaporization is computed so that the exact variation of enthalpy of the fuel between its liquid state at initial temperature and its hot vapor state is eventually transferred to the gas phase. So the kinetics of vaporization is a source of transient uncertainty but the model is thermodynamically consistent.

\subsection{Chemistry model}

For the chemistry of ignition and combustion, we use the 2-step mechanism by Hakim et al. [63]. It has been calibrated using Bayesian inference to accurately predict autoignition over the ranges of conditions (pressure, temperature, equivalence ratio) relevant to the Spray A case. The mechanism accounts for the incomplete oxidation of a Diesel surrogate, $\mathrm{n}$-dodecane,

$$
\begin{aligned}
\mathrm{C}_{12} \mathrm{H}_{26}+\frac{25}{2} \mathrm{O}_{2} & \rightarrow 12 \mathrm{CO}+13 \mathrm{H}_{2} \mathrm{O} \\
\mathrm{CO}+\frac{1}{2} \mathrm{O}_{2} & \rightleftharpoons \mathrm{CO}_{2}
\end{aligned}
$$

and allows the Arrhenius pre-exponential factor of the first reaction to vary with the local fresh gas conditions. The calibration aims at the autoignition delay time given by a skeletal mechanism [64] in homogeneous reactors for temperatures $T_{0}$ ranging from $600 \mathrm{~K}$ to $1200 \mathrm{~K}$ and equivalence ratios, $\phi$, between 0.5 and 4 . These ranges of interest were extracted from a Spray A simulation [54] that characterized the mixture in the flammable region just prior to ignition. Since the present mechanism has not yet been optimized for flame propagation, we take advantage of the fact that ignition and flame propagation occur in two separate ranges of temperatures [65]. Accordingly, a well-mixed turbulent combustion model is used, which is acceptable for the slow processes that lead to auto-ignition but will overestimate flame propagation.

\subsection{Numerical methods}

Euler-Euler modeling of sprays is known for requiring dedicated numerical methods and only recent progress has enabled its use [16]. A novel semi-Lagrangian transport scheme has been devised by Doisneau et al. [18] together with a splitting method that efficiently enforces strong coupling between the two phases. This scheme achieves realizable transport of the moments, whatever the size and velocity reconstructions are, so that no clipping is needed. This guarantees both accuracy and robustness as well as ease in handling the code and setting the configurations.

In the context of operator splitting, the transport part of the gas phase reduces to the Navier-Stokes system, for which a staggered explicit time integration method (RK4) with $3^{\text {rd }}$ order fluxes is chosen [66]. This allows one to account accurately for both the convective and acoustics structures in the flow. To preserve the coupling between the two phases, the splitting time step is chosen to be equal to the gas phase time step [27]. Even though the coupling is fast when loading is high, the fine spatial resolution brings $\Delta t$ below $10^{-8} \mathrm{~s}$ so the CFL remains the harshest stability constraint, together with being the criterion of accuracy of the splitting. The mass, momentum, and heat transfers are then integrated explicitly. This model 
and methodology have been implemented in the RAPTOR LES solver developed by Oefelein [66].

\section{Numerical results}

To demonstrate the model, we consider conditions from the ECN Spray A case [32]. Here n-dodecane $\left(\mathrm{C}_{12} \mathrm{H}_{26}\right.$, $\left.\rho_{l}=702 \mathrm{~kg} / \mathrm{m}^{3}, T_{l}=363 \mathrm{~K}\right)$ is injected as a round jet of liquid drops at a speed of $\boldsymbol{u}_{l}\left(t, \boldsymbol{x}_{0}\right)=600 \mathrm{~m} / \mathrm{s}$ from a single injector $\left(d_{\text {inj }}=90 \mu \mathrm{m}\right)$ into a chamber $\left(T_{g}=900 \mathrm{~K}\right.$, $\left.P_{g}=6 \mathrm{MPa}\right)$.

\subsection{Momentum coupling}

We perform a 7 cell $/ d_{i n j}$ non-reacting computation. The chamber is filled with pure nitrogen $\left(\mathrm{N}_{2}\right.$ of density $\rho_{g}=$ $22.4 \mathrm{~kg} / \mathrm{m}^{3}$ ). As an approximation, a laminar plug-flow profile is used. The geometry is simplified to a box of dimensions $9.6 \times 3.2 \times 3.2 \mathrm{~mm}^{3}$ discretized as a cartesian mesh of $768 \times 256 \times 256$ cells. To study momentum coupling, no heating or evaporation is considered. For the drag force characteristic time, the equivalent drop radius is taken to be $r=2 \mu \mathrm{m}$, which leads to coupling times that are slightly above the splitting and the convection timesteps, $\Delta t=8 \mathrm{~ns}$. This small size is used to test the overall quality of the numerical strategy in the presence of stiff transfer terms and strong coupling. It also corresponds to the possible droplet sizes produced by Spray A atomization as reported by X-ray and microscopy measurements.

Fig. 2 is a snapshot of the jet near-nozzle region shortly after start of injection. We extract time-averaged and RMS values of gas and spray quantities from a slice at $x=50 d_{\text {inj. }}$ After azimuthal averaging, the radial statistics presented in Fig. 3 appear smooth. Fully checking convergence would require an analysis over longer times. Still, this demonstrates the ability of the Eulerian spray model to capture additional scales compared to traditional injection LES, without relying on stochastic models or dubious undersampling with the random seed as a realization parameter.

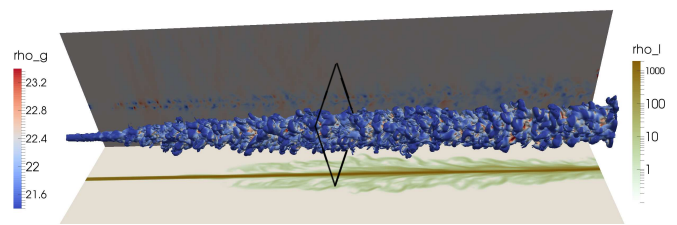

Figure 2: Non-reacting jet at $t_{0}=32 \mu$ s showing the position of the $x=$ $50 d_{\text {inj }}$ slice. Isocontour of vorticity norm colored by gas axial velocity (blue: 0; red: $100 \mathrm{~m} / \mathrm{s}$ ); Back plane: Gas pressure in the vertical center plane; Bottom plane: Spray mass concentration in the horizontal center plane.

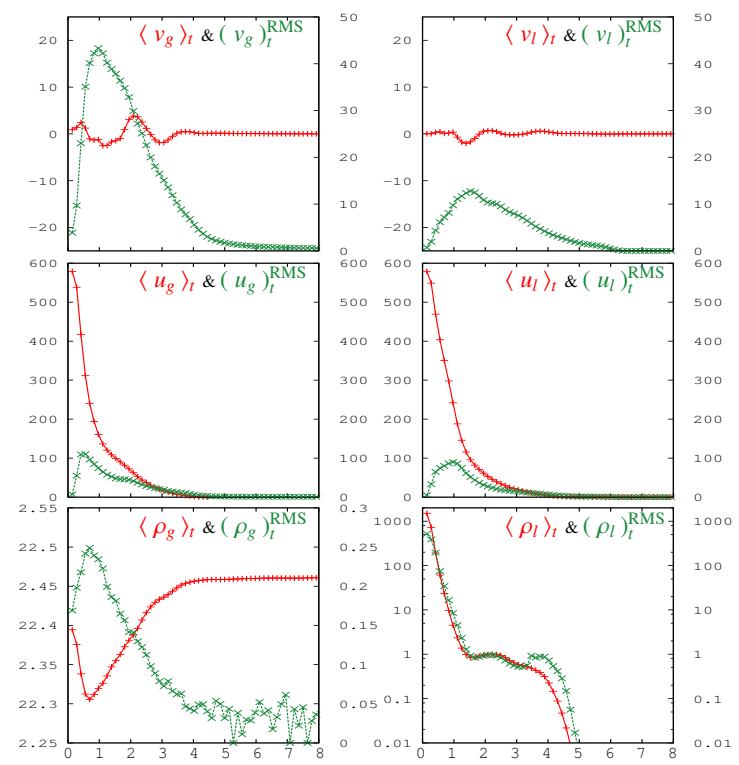

Figure 3: Time averages $(+)$ and RMS $(\times)$ along a radius (azimuthal average at $x=50 d_{\text {inj }}$ ). Radial scale is in units of $d_{\text {inj }}$. Azimuthal components $w_{g / l}$ (not shown) behave like $v_{g / l}$.

\subsection{Autoignition}

A 4 cell $/ d_{i n j}$ reacting computation is then performed. According to Spray A specifications, the chamber is filled with a mixture of the burnt gases left by the preheating of molar fractions $\mathrm{O}_{2}: 15.00 \%, \mathrm{~N}_{2}: 75.15 \%, \mathrm{CO}_{2}: 6.22 \%$, $\mathrm{H}_{2} \mathrm{O}: 3.62 \%$, and density $\rho_{g}=22.8 \mathrm{~kg} / \mathrm{m}^{3}$. A laminar plug-flow profile is used with a realistic velocity ramp to match the measured rate of injection. The geometry is simplified to a box of size $27 \times 5.4 \times 5.4 \mathrm{~mm}^{3}$ discretized as a cartesian mesh of $1200 \times 240 \times 240$ cells. We present in Fig. 4 an instantaneous snapshot of the reacting jet after autoignition. This shows the ability of the method to capture the mixing and combustion occurring in the liquid-gas shear layer. Improved models; e.g., for polydispersity and spray combustion, and quantitative validation are still to be considered.

\section{Conclusions}

Spray injection is a multiscale problem. But today's applied simulations can only tackle the coarsest, chamber level. We have analyzed various two-phase flow formalisms and we have shown that the Eulerian spray formalism coupled to Large Eddy Simulation is well suited to capture chamber and mixing layer flow scales. We have developed a framework and numerical methods to make this description operative to describe a fuel liquid core and spray at typical engine conditions and we have shown how 
it performs on a well-documented spray with realistic autoignition.

Accurately describing polydispersity is a key capability provided the sensitivity of spray physics to droplet sizes. Future work is i) to take advantage of a MultiFluid strategy to describe atomization with more fidelity on the droplet sizes [27, 60], and ii) to select and implement the velocity closure that is relevant to inertia of each droplet sizes [29]. To alleviate the arbitrary aspect of our size boundary condition we plan to prescribe a sectional discretization of the size outcome of spray A atomization [18], as computed with a CLSVOF method [3]. With these, our strategy will still not account for i) liquid volume occupancy, and ii) large-scale surface tension effects. Comparisons to experimental results should then validate if the physics of high inertia and high Weber number allows us to neglect i) and ii).

Over time, we believe that a spray-resolved approach will provide the needed fidelity to devise transient, local, realization-dependent quantities of interest and to perform new-generation spray studies; e.g., on ignition variability.

\section{Acknowledgements}

Support by Sandia National Laboratories' Laboratory Directed Research and Development (LDRD) program is also gratefully acknowledged. The authors also thank Layal Hakim for providing the two-step chemistry model. Sandia National Laboratories is a multi-program laboratory managed and operated by Sandia Corporation, a wholly owned subsidiary of Lockheed Martin Corporation, for the U.S. Department of Energy's National Nuclear Security Administration under contract DE-AC0494AL85000.

\section{References}

[1] T. Menard, S. Tanguy, A. Berlemont, Int. J. Multiph. Flows 33 (2007) 510-524.

[2] O. Desjardins, H. Pitsch, Atom. Spr. 20 (2010).

[3] M. Arienti, M. Sussman, SAE Tech. Paper 01-1853 (2015).

[4] M. Bode, F. Diewald, D. O. Broll, J. F. Heyse, V. Le Chenadec, H. Pitsch, SAE Tech. Paper 01-1427 (2014).

[5] S. El Tahry, D. Haworth, J. Prop. Power 8 (1992) 1040-1048.

[6] D. Haworth, K. Jansen, Computers \& fluids 29 (2000) 493-524.

[7] K. Liu, D. Haworth, Flow. Turb. Comb. 85 (2010) 279-307.

[8] K. Liu, D. C. Haworth, X. Yang, V. Gopalakrishnan, Flow. Turb. Comb. 91 (2013) 373-403.

[9] J. Abraham, L. M. Pickett, Atom. Spr. 20 (2010).

[10] P. Senecal, E. Pomraning, K. Richards, S. Som, SAE Tech. Paper 01-1083 (2013).

[11] J. Shinjo, A. Umemura, Proc. Comb. Inst. 34 (2013) 1553-1560.

[12] Z. Li, A. Banaeizadeh, F. A. Jaberi, J. Fluid Mech. 760 (2014) 243277.
[13] F. Doisneau, M. Arienti, J. C. Oefelein, in: $13^{\text {th }}$ ICLASS, Tainan, Taiwan, pp. 1-12.

[14] J. Shinjo, J. Xia, A. Umemura, Proc. Comb. Inst. 35 (2015) 15951602.

[15] M. Bode, T. Falkenstein, H. Pitsch, T. Kimijima, H. Taniguchi, T. Arima, in: $13^{\text {th }}$ ICLASS, Tainan, Taiwan, pp. 1-12.

[16] S. de Chaisemartin, Polydisperse evaporating spray turbulent dispersion: Eulerian model and numerical simulation, Ph.D. thesis, Ecole Centrale Paris, 2009.

[17] O. Emre, D. Kah, S. Jay, Q.-H. Tran, A. Velghe, S. De Chaisemartin, R. Fox, F. Laurent, M. Massot, Atomization and Sprays 25 (2015).

[18] F. Doisneau, M. Arienti, J. C. Oefelein, A semi-Lagrangian transport method for kinetic problems: application to dense multi-phase flows, 2016. Accepted for publication in J. Comp. Phys.

[19] C. Rowley, Int. J. Bifurc. Chaos 15 (2005) 997-1013.

[20] A. Quarteroni, G. Rozza, Reduced Order Methods for Modeling and Computational Reduction, volume 9, Springer, 2014.

[21] G. A. Bird, Molecular gas dynamics and the direct simulation of gas flows, volume 42, Oxford Science Publications, 1994.

[22] F. Laurent, M. Massot, Comb. Th. Model. 5 (2001) 537-572.

[23] O. Thomine, Développement de méthodes multi-échelles pour la simulation numérique des écoulements réactifs diphasiques, Ph.D. thesis, Université de Rouen, 2011.

[24] S. Subramaniam, Prog. Ener. Comb. Sc. 39 (2013) 215-245.

[25] F. Salehi, M. J. Cleary, A. R. Masri, SAE Tech. Paper 2016-01-0859 (2016).

[26] F. Doisneau, J. Dupays, A. Murrone, F. Laurent, M. Massot, C. R. Mec. 341 (2013) 44-54. Special issue "Combustion, spray and flow dynamics for aerospace propulsion".

[27] F. Doisneau, A. Sibra, J. Dupays, A. Murrone, F. Laurent, M. Massot, J. Prop. Power 30 (2014) 727-747.

[28] J. Reveillon, F. Demoulin, J. Fluid Mech. 583 (2007) 273-302.

[29] A. Vié, F. Doisneau, M. Massot, Comm. Comp. Phys. 17 (2015) $1-46$.

[30] C. Yuan, R. Fox, J. Comp. Phys. 230 (2011) 8216-8246.

[31] F. A. Williams, Phys. Fluids 1 (1958) 541-545.

[32] M. Bardi, R. Payri, L.-M. Malbec, G. Bruneaux, L. M. Pickett, J. Manin, T. Bazyn, C. Genzale, Atom. Spr. 22 (2012)

[33] N. Bharadwaj, C. J. Rutland, Atom. Spr. 20 (2010).

[34] S. Som, S. Aggarwal, Comb. Flame 157 (2010) 1179-1193.

[35] S. Som, A. I. Ramirez, D. E. Longman, S. K. Aggarwal, Fuel 90 (2011) 1267-1276.

[36] M. Bolla, T. Gudmundsson, Y. M. Wright, K. Boulouchos, SAE Int. J. Eng. 6 (2013) 1249-1261.

[37] S. Bhattacharjee, D. C. Haworth, Comb. Flame 160 (2013) 20832102.

[38] A. Maghbouli, T. Lucchini, G. D’Errico, A. Onorati, Appl. Therm. Eng. 91 (2015) 901-912.

[39] S. Ayyapureddi, U. Egüz, C. Bekdemir, L. Somers, L. De Goey, in: $12^{\text {th }}$ ICLASS.

[40] A. Irannejad, A. Banaeizadeh, F. Jaberi, Comb. Flame 162 (2015) 431-450.

[41] T. Lucchini, G. d'Errico, D. Ettorre, G. Ferrari, SAE Tech. Paper 01-1971 (2009).

[42] G. D'Errico, T. Lucchini, F. Contino, M. Jangi, X.-S. Bai, Comb. Th. Model. 18 (2014).

[43] C. Bekdemir, L. Somers, L. de Goey, J. Tillou, C. Angelberger, Proc. Comb. Inst. 34 (2013) 3067-3074.

[44] J. Tillou, J.-B. Michel, C. Angelberger, D. Veynante, Comb. Flame 161 (2014) 525-540.

[45] Q. Xue, S. Som, P. K. Senecal, E. Pomraning, Atom. Spr. 23 (2013).

[46] P. K. Senecal, E. Pomraning, K. Richards, S. Som, J. Ener. Resourc. Tech. 136 (2014) 012204.

[47] B. Hu, S. Banerjee, K. Liu, S. Rajamohan, J. M. Deur, Q. Xue, 
S. Som, E. Pomraning, P. K. Senecal, ASME Tech. Paper ICEF1033 (2015)

[48] C. Gong, M. Jangi, T. Lucchini, G. D’Errico, X.-S. Bai, Flow. Turb. Comb. 93 (2014) 385-404

[49] C. Gong, M. Jangi, X.-S. Bai, Appl. Energ. 136 (2014) 373-381.

[50] Y. Pei, E. R. Hawkes, S. Kook, G. M. Goldin, T. Lu, Comb. Flame 162 (2015) 2006-2019.

[51] A. Wehrfritz, V. Vuorinen, O. Kaario, M. Larmi, Atom. Spr. 23 (2013).

[52] P. K. Senecal, E. Pomraning, Q. Xue, S. Som, S. Banerjee, B. Hu, K. Liu, J. Deur, J. Eng. Gas Turb. Power 136 (2014) 111504.

[53] F. Doisneau, M. Arienti, J. C. Oefelein, in: $9^{\text {th }}$ US National Combustion Meeting, Cincinnati, USA, pp. 1-11.

[54] G. Lacaze, A. Misdariis, A. Ruiz, J. Oefelein, Proc. Comb. Inst. (2015).

[55] P. Senecal, E. Pomraning, K. Richards, S. Som, ASME Tech. Paper ICEF-92043 (2012) 697-710.

[56] J. Chesnel, T. Ménard, J. Reveillon, F. Demoulin, Atom. Spr. 21 (2011) 41-67.

[57] J. McCaslin, C. Y. Goh, O. Desjardins, in: 26th ILASS

[58] M. Garcia, Développement et validation du formalisme EulerLagrange dans un solveur parallèle non-structuré pour la simulation aux grandes échelles., Ph.D. thesis, Institut National Polytechnique de Toulouse, 2009.

[59] C. Chalons, M. Massot, A. Vié, Multiscale Modeling \& Simulation 13 (2015) 291-315.

[60] F. Laurent, F. Laurent, A. Sibra, Comm. Comp. Phys. (2016) .

[61] D. Kah, O. Emre, Q. Tran, S. Chaisemartin, S. Jay, F. Laurent, M. Massot, Int. J. Multiph. Flows 71 (2015) 38-65.

[62] F. Laurent, A. Sibra, F. Doisneau, Submitted to Comm. Comp. Phys. (2015).

[63] L. Hakim, G. Lacaze, M. Khalil, H. Najm, J. Oefelein, ASME Tech. Paper ICEF-1120 (2015).

[64] K. Narayanaswamy, P. Pepiot, H. Pitsch, Comb. Flame 161 (2014) 866-884.

[65] A. Misdariis, O. Vermorel, T. Poinsot, Proc. Comb. Inst. 35 (2015) 3001-3008.

[66] J. C. Oefelein, Prog. Aerosp. Sc. 42 (2006) 2-37. 
Table 1: Nomenclature of various models for transport in physical space. Velocity closures include PGD: pressureless gas dynamics [16], IG/AG/MG: isotropic/anisotropic/multi-Gaussian [29], CQMOM: conditional quadrature method of moments [30].

\begin{tabular}{l|c|c|c|l|l}
\hline & Formalism & Hyperbolicity & Compressibility & Pros & Cons \\
\hline DPS & Lagrangian & yes, linear & hyper & deterministic & $\begin{array}{l}\text { cost, initial/boundary conditions } \\
\text { hinetic }\end{array}$ \\
hyph dimensionality (7D/9D)
\end{tabular}

Table 2: Grid resolution $d_{i n j} / \Delta x$ of current spray A simulations. Number of particles is given in millions of parcels (Mp) for DSMC (if specified) to indicate sampling resolution. Subgrid models include Turbulent transport (RANS, LES), Atomization (LS: Line-Source, HG: Huh-Gosman, RD: ReitzDiwakar, KHACT: Aerodynamics Cavitation Turbulence), Secondary Break-up (HF: Hsiang-Faeth, PE: Pilch-Erdmann, MPI, KH: Kelvin-Helmholtz, RT: Rayleigh-Taylor, ETAB: Extended Taylor Analogy Break-up), and Turbulent Combustion (WM: Well Mixed, TPDF: Transported Probability Density Function, CMC: Conditional Moment Closure, MMC: Multiple Mapping Conditioning, CCM: Chemistry Coordinate Mapping, mRIF: multiple Representative Interactive Flamelets, FMDF: Filtered Mass Density Function, FGM: Flame Generated Manifold). Simultaneous (alternate) use of subgrid models is marked with dash (slash).

\begin{tabular}{|c|c|c|c|}
\hline Authors & Method for liquid & $d_{i n j} / \Delta x$ & Subgrid models \\
\hline Bharadwaj and Rutland [33] & DSMC & 0.18 & LES \\
\hline Som and Aggarwal [34], Som et al. [35] & DSMC & 0.18 & KHACT-KHRT-2DRANS \\
\hline Bolla et al. [36] & DSMC & 0.18 & HG/RD-CMC-2DRANS \\
\hline Bhattacharjee and Haworth [37] & DSMC (0.05 Mp) & 0.30 & RD-TPDF-2DRANS \\
\hline Abraham and Pickett [9] & DSMC & 0.30 & LS-2DRANS \\
\hline Maghbouli et al. [38] & DSMC & 0.36 & HG/KH-RANS \\
\hline Ayyapureddi et al. [39] & DSMC & 0.36 & HG/RD-HF/PE/MPI/KH-FGM-2DRANS \\
\hline Irannejad et al. [40] & DSMC (120 Mp) & 0.50 & FMDF-LES \\
\hline Abraham and Pickett [9] & Eulerian (VLS) & 0.88 & 2DRANS \\
\hline Lucchini et al. [41], D'Errico et al. [42] & DSMC & 0.90 & HG-WM/mRIF-2DRANS \\
\hline Bekdemir et al. [43], Tillou et al. [44] & Eulerian (downstream inj.) & 1.1 & TPDF-LES (fuel is heptane) \\
\hline Xue et al. [45], Senecal et al. [10, 46], Hu et al. [47] & DSMC (0.8 Mp) & $<1.4$ & RD-KHRT-LES \\
\hline Gong et al. $[48,49]$ & $\operatorname{DSMC}(0.5 \mathrm{Mp})$ & 1.4 & HG-WAVE-CCM-LES \\
\hline Abraham and Pickett [9] & Equivalent Gas & 2.0 & 2DRANS \\
\hline Pei et al. [50] & DSMC & 2.0 & TPDF-2DRANS \\
\hline Wehrfritz et al. [51] & DSMC & 2.2 & KHRT/ETAB-LES \\
\hline Senecal et al. [52] & DSMC (21 Mp) & $<2.9$ & RD-KHRT-LES \\
\hline Present (autoignition) & Eulerian & 4.0 & WM-LES \\
\hline Salehi et al. [25] & Equivalent Gas & 6.0 & MMC-LES \\
\hline Doisneau et al. $[18,53]$, present (mom. coupling) & Eulerian & 7.0 & LES \\
\hline Lacaze et al. [54] & NS (Real Gas) & 30 & LES \\
\hline Arienti and Sussman [3] & NS (+ interface capt.) & 30 & None \\
\hline Bode et al. [4] & NS (+ interface capt.) & 43 & None \\
\hline
\end{tabular}

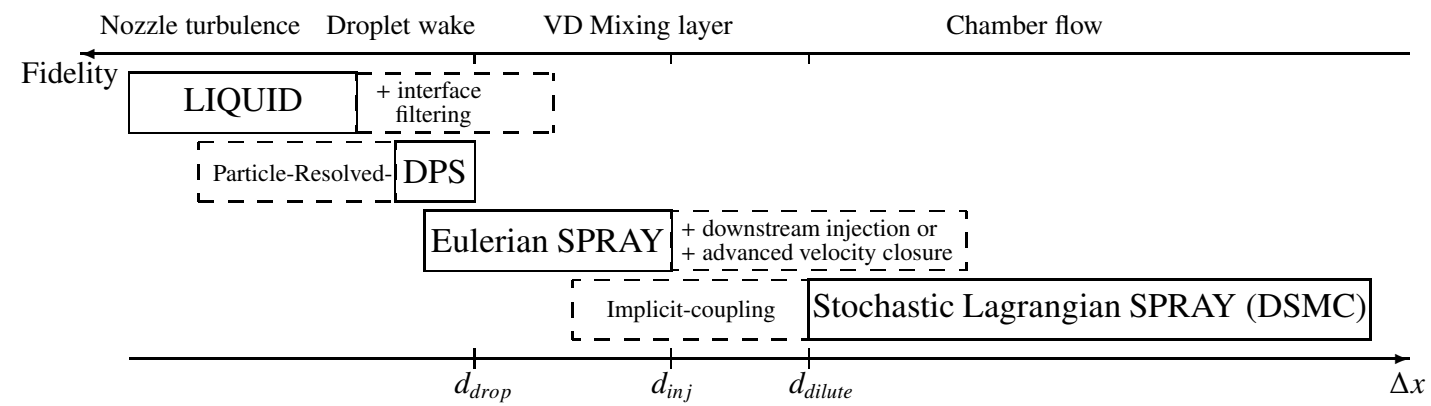

Figure 1: Validity of liquid phase approaches as a function of spatial resolution $\Delta x$ of the Eulerian grid used for the carrier gas. Dashed boxes show possible developments to extend validity domains of approaches. 


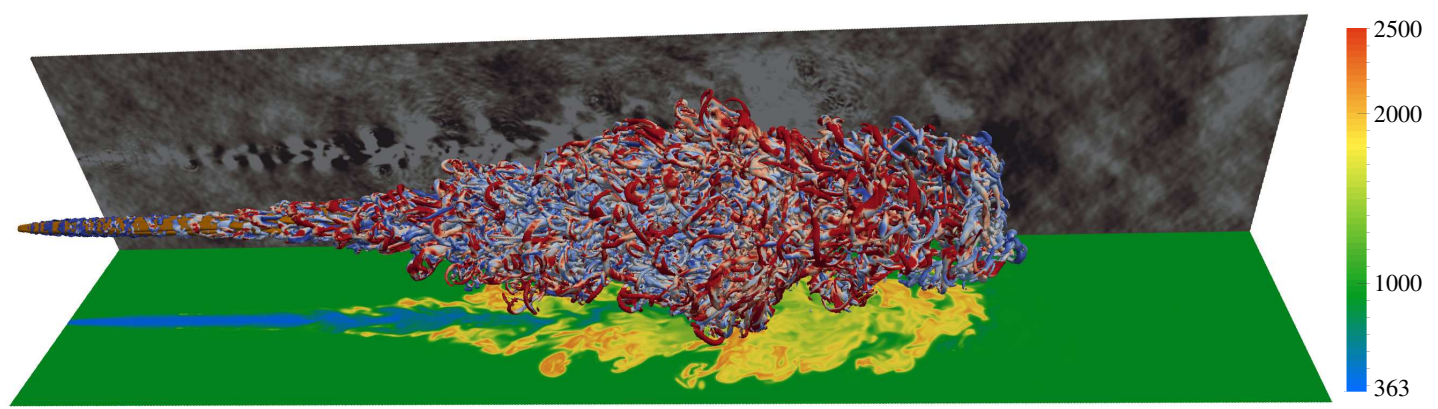

Figure 4: Reacting jet at $t_{1}=150 \mu \mathrm{s}$. Isocontour of Q-criterion colored by CO mass fraction (blue: $0 \%$; red: $0.5 \%$ ); Back plane: Gas pressure in the vertical center plane; Bottom plane: Gas temperature in the horizontal center plane. 